\title{
NUMERICAL PREDICTION OF A LARGE BUBBLE BEHAVIOR IN WALL TURBULENT FLOW
}

\author{
SANGWON KIM ${ }^{1}$ AND NOBUYUKI OSHIMA ${ }^{2}$ \\ ${ }^{1}$ Mechanical and Space Engineering, Hokkaido University \\ Hokkaido, Japan 060-8626 \\ swkim5834@eis.hokudai.ac.jp \\ ${ }^{2}$ Mechanical and Space Engineering, Hokkaido University \\ Hokkaido, Japan 060-8626 \\ oshima@eng.hokudai.ac.jp
}

Key words: Two phase flow, Turbulence, Drag reduction, Bubble deformation.

\begin{abstract}
Air lubrication systems for underwater applications have gained great popularity in recent years. Large bubbles, around $\mathrm{We} \geq 100$, show a large area of drag reduction than a small and microbubble, especially for large scale flow problems such as ship surface. However, it is hard to maintain their shape and prone to deform in a turbulent flow. In order to understand and control the drag reduction mechanism by the large bubble injection, it is necessary to know the behavior of large bubbles in the turbulent boundary layer and their interaction with the skin friction. In this study, a solver "interIsoFoam" for two-phase flow of an open-license software "OpenFOAM" is applied for an LES of turbulent channel flow with a large bubble, the gas-liquid interface of which is directly captured by improved VOF method. In a recent publication [1], we presented the numerical procedure of how to inject the large bubble on turbulent channel flow. The research objectives in this study are investigation on flow change of the horizontal channel flow included large bubble. From this observation, understanding large bubbles characteristic such as the surrounding flow phenomena was observed.
\end{abstract}

\section{INTRODUCTION}

As growing the attention of global warming all around the world, the IMO released the requirement on the ship's greenhouse gas emission. To comply with increasing EEDI Phase 3 levels, the air lubrication system is one of the technologies to achieve it and developed to meet the industry need [2]. This system can be categorized into two main categories, the microbubble method started by McCormick and Bhattacharyya [3] and the air-film method (Fukuda et al., [4][5]). Recently, the above-mentioned two methodologies have the same issue on a decrease of drag reduction performance from downstream because of the large bubble. Meanwhile, Murai et al. [6][7] reported the drag reduction mechanism for these bubbles (10$50 \mathrm{~mm}$ ) and motivated a turning point in the drag reduction of the large bubble, as a new material for drag reduction. In addition, The advantage is that supplying a large bubble is much easier than generating a high flow rate of microbubbles or stabilizing air films with a wide area. Oishi and Murai [8] investigated and depicted detailed characteristics related to 
drag reduction by measuring the single large bubble. Kim et al. [1] established a numerical procedure how to inject a large bubble on turbulent channel flow and investigated the behavior of a large bubble with wall turbulent flow. Interestingly, the vortical structures showed between the bubble and upper wall on the liquid film and secondary flow. In this study, a numerical simulation of the turbulent channel flow containing a large bubble is carried out, we investigate the flow field around the bubble and the mechanism of generating vortical structure.

\section{NUMERICAL METHOD}

\subsection{Governing equations}

In this study, the flow is considered incompressible and the fluids are considered Newtonian. The governing equations in this study for continuity and momentum equation can be expressed by Eq. (1) and (2), respectively, and the mass conservation equation is presented in Eq. (3).

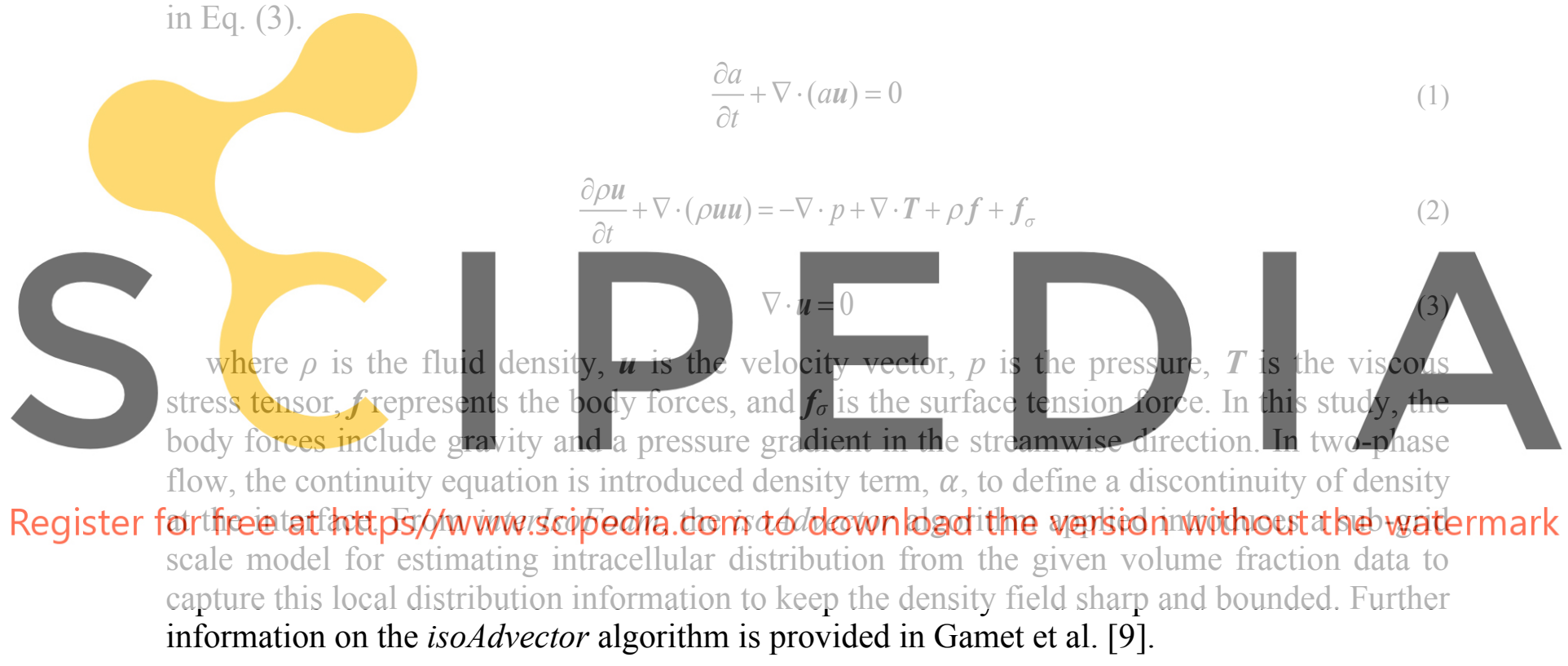

\subsection{Numerical setup}

The numerical condition and method in this study were reported by Kim et al. [1] based on Oishi and Murai [8], shown in Tables 1 and 2. In addition, the geometrical configuration of the computational domain is presented in Fig. 1, the size of the domain is $10 \mathrm{~mm}$ high $(\mathrm{H}=$ 2h), $75 \mathrm{~mm}$ wide, and $200 \mathrm{~mm}$ long between two horizontal walls. The flow is driven from the whole region by a mean pressure gradient. In addition, injecting condition is located $100 \mathrm{~mm}$ from upstream with a rectangular shape, at the upper wall. Table 3 shows the procedure of numerical simulation is divided into 3 stages to inject and maintain a large bubble successfully, and the detail is described in Kim et al [1]. In all cases, bubbles are added after turbulent channel flow, which is generated by pimpleFoam of OpenFOAM, then bubble simulation is performed through interIsoFoam. 


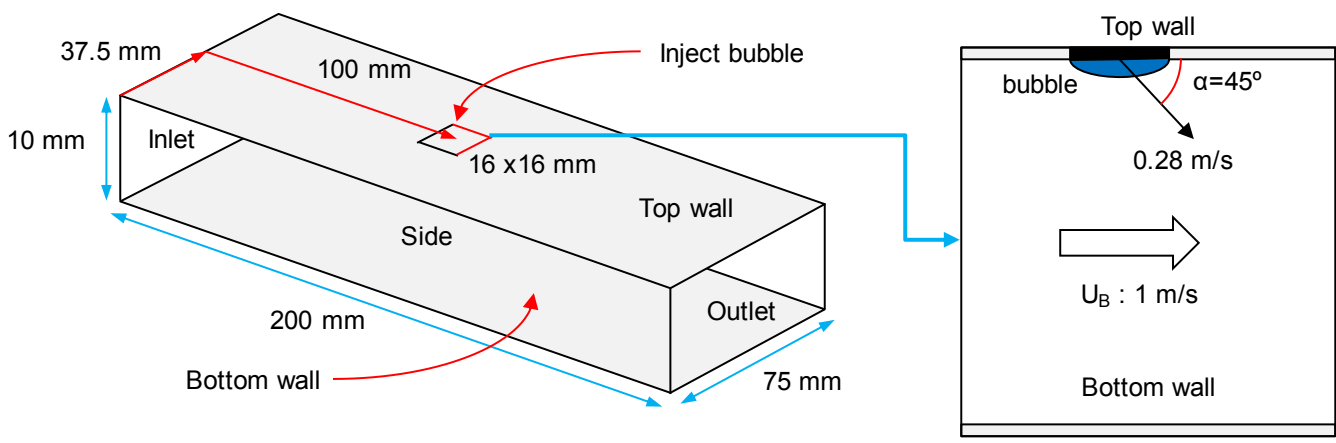

Figure 1: Computational domain and Boundary condition [9].

Table 1: Numerical condition [1].

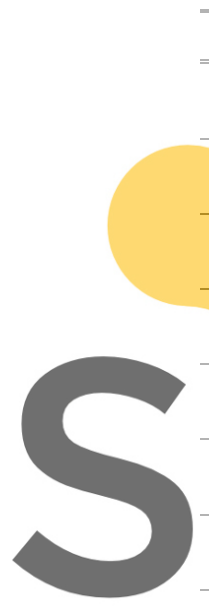

$10 \times 75 \times 200$

Water $\left(17^{\circ} \mathrm{C}\right)$

Condition

$\left(\mathrm{kg} / \mathrm{m}^{3}\right)$

$v\left(\mathrm{~m}^{2} / \mathrm{s}\right)$

$U_{\text {mean }}(\mathrm{m} / \mathrm{s})$

$\operatorname{Fr}(-)$

$\operatorname{Re}(-)$

$R e_{\tau}(-)$
998.7

\section{$1.08 \times 10^{-6}$}

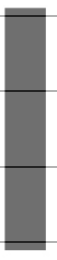

Present CFD
Table 2: Numerical method [1].

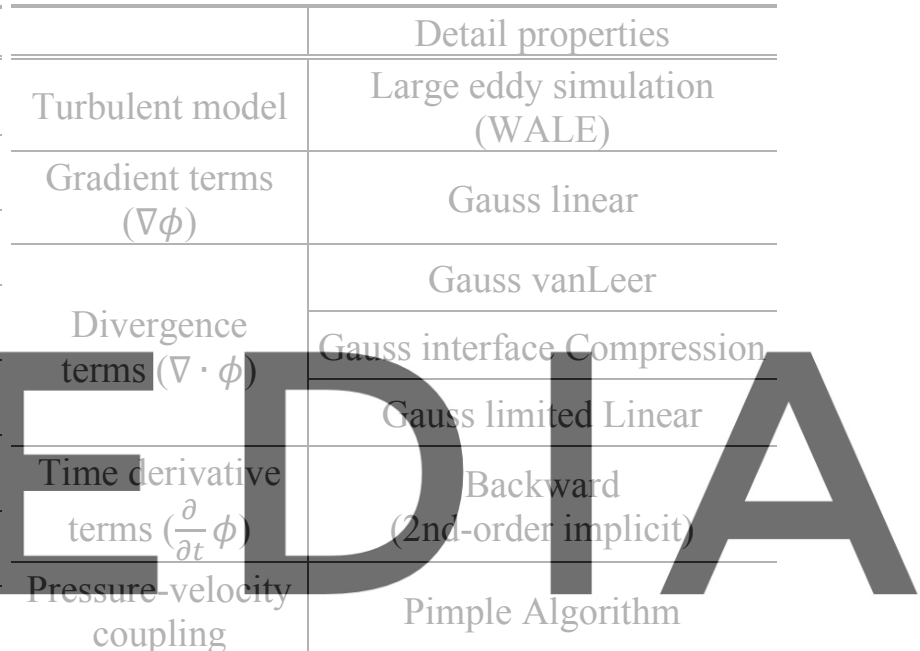

Register for free at https//www.scipedia.com to download the version without the watermark

Table 3: Boundary condition in each stage. [1]

\begin{tabular}{c|ccccc}
\hline & Stage 1 & & Stage 2 & Stage 3 \\
\hline \hline Solver & PimpleFoam & $\rightarrow$ & InterIsoFoam & InterIsoFoam \\
\hline Inlet & Cyclic with Outlet & $\rightarrow$ & Fixedvelocity & $\rightarrow$ & Cyclic with Outlet \\
\hline Outlet & Cyclic with Inlet & $\rightarrow$ & InletOutlet & $\rightarrow$ & Cyclic with Inlet \\
\hline Side & & \multicolumn{2}{c}{ Cyclic between Left and Right } \\
\hline Bottom & Noslip & $\rightarrow$ & \multicolumn{2}{c}{ Contact angle $\left(1^{\circ}\right)$} \\
\hline Top & Noslip & $\rightarrow$ & $\begin{array}{c}\text { Codestream for } \\
\text { Injection of bubble }\end{array}$ & $\rightarrow$ & Contact angle $\left(1^{\circ}\right)$ \\
\hline
\end{tabular}

\section{NUMERICAL RESULTS AND DISCUSSION}

The general properties and shape of bubbles are summarized in Table. 4 and Fig. 2. In the table, the diameter is calculated by the bubble's volume, which is evaluated from the numerical simulation. In addition, Weber number, which characterizes the deformation of the bubble, is defined as: 


$$
W e=\frac{\rho U_{b}^{2} d}{\sigma}
$$

In the case of shape factor, it is defined as $l_{1} / l_{2}, l_{1} / b, l_{2} / b$, where $l_{1}$ and $l_{2}$ are streamwise and spanwise length, $b$ is the height of the bubble, respectively. In the case of the bubble shape, The interface stretched to streamwise and spanwise directions, and the thickness of the liquid film gradually decreased in the direction of the rear of the bubble. The comparison of bubble shape as the series of samples was explained in Kim et al. [1].

Table 4: Summary of bubble parameters. [1]
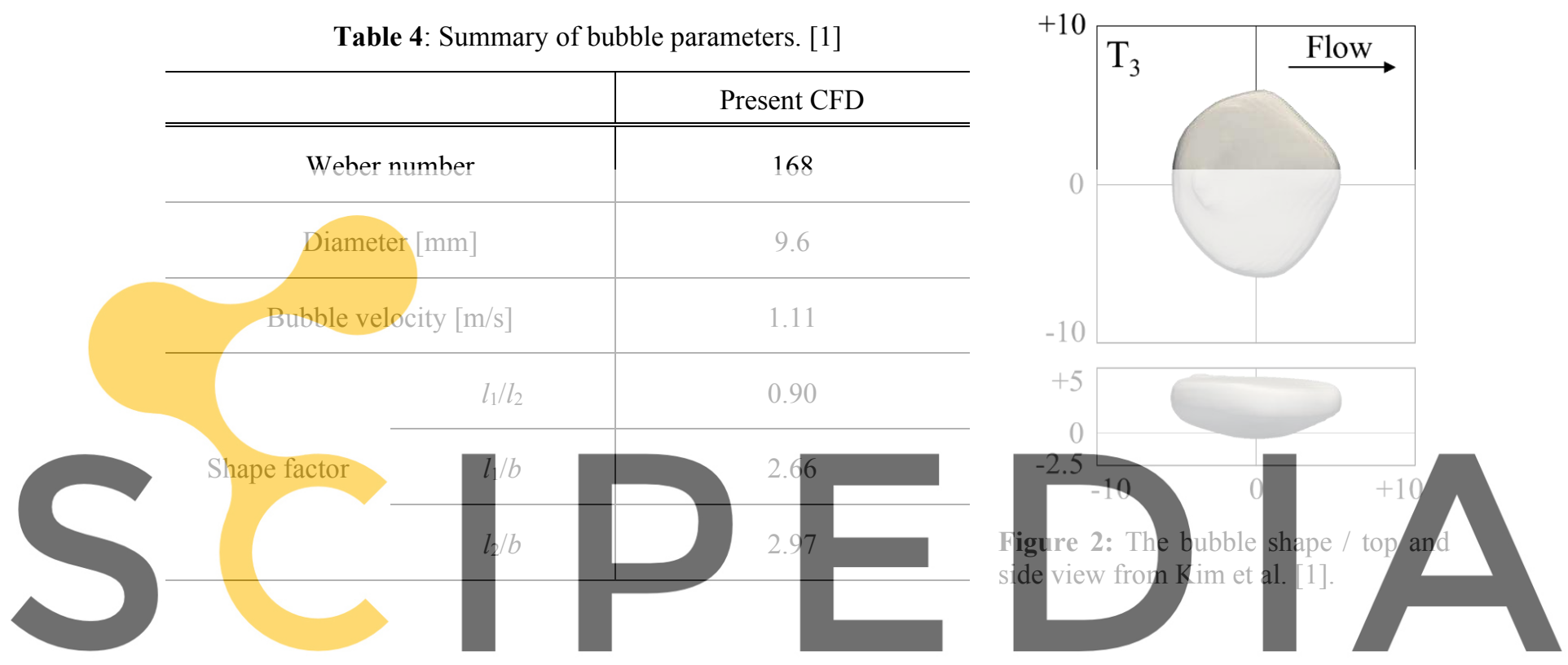

(a)

Register for free at https//www.scipedia.com to download the version without the watermark

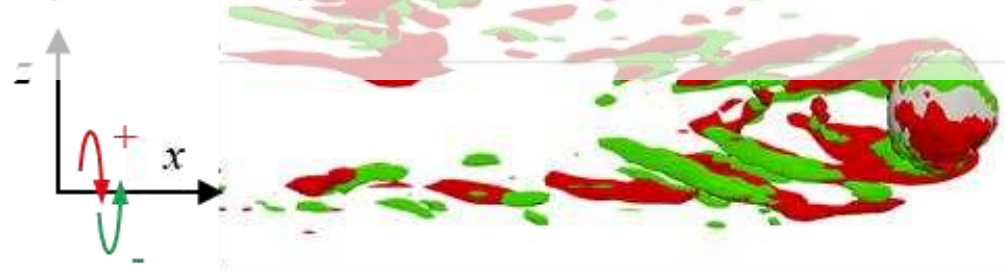

(b)

\section{Starting point of the vortices}

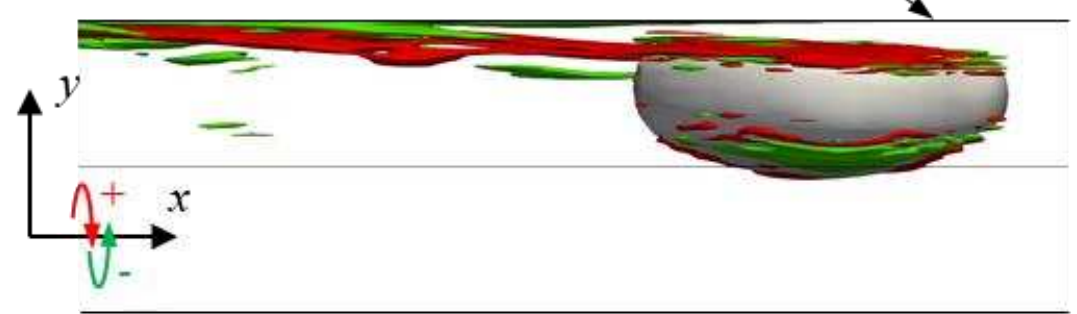

Figure 3: Snapshots on Iso-surface of vorticity streamwise component; (a) Bottom view, (b) Side view from Kim et al. [1]. 
Fig. 3 show the iso-surface of vorticity in streamwise component around the bubble. The vorticity values are \pm 330 , visualized from the side and bottom view. The counter-rotating vortices are developed around the centroid of the bubble. Simultaneously, the counter-rotating vortices are also developed at the same side, but the size of the pairs is unequal as shown in Fig. 3(a). From Fig.3(b), the origin of these vortices can be observed from the wall and bubble interface. These vortices spread widely then separate with forming horseshoe vortices. In the horseshoe vortices of the same side, the positions of these structures are corresponding with each other and the tilting angle of vortices is $\pm 25^{\circ}$. The sequence of generating these vortices was discussed in detail by Kim et al. [1]. This means that the current size of the bubble turns the flow condition below the buffer layer into the turbulent regime. The reason why vortices are generated can be explained by the instantaneous velocity from the section view of the bubble. Figs. 4 and 5 show the velocity vector contours on the front and side view and obtained from the centroid of the bubble. The vector is originated from the relative velocity, expressed as:

$$
\boldsymbol{u}(x, y, z)=\boldsymbol{u}_{0}(x, y, z)-\boldsymbol{u}_{\text {mean }}(x, y, z), \boldsymbol{u}_{\text {mean }}(x, y, z)=(1,0,0)
$$

where $\boldsymbol{u}_{0}$ is velocity vector, $\boldsymbol{u}_{\text {mean }}$ is target velocity of the pressure gradient in the streamwise direction. Fig. 4 shows the velocity vector contour from the front view, the vector is obtained from $y$ and $z$ components. we can clearly observe four vortexes inside the bubble. Based on the rotating direction, the pair of vortices at the same height which rotates in counter-direction caused instantaneous velocity heads outside of the bubble, while the pair of vortices at the same width generated instantaneous velecity heads center of the bubble. This
feature also shows from Fig.5 which visualized the vector contour of $x$ and $y$ components
from the side view. Thus, we can understand the reason why large bubble extends streamwise
and spanwise direction that wall-normal direction. In the case of the velocity contour at the
liquid film, the velocity in the spanwise direction head for outside as shown in Fig. 4(a). In addition, its magnitude value increase as the location change to outside in the spanwise

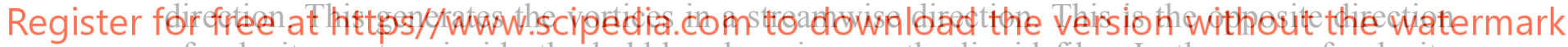
of velocity vectors inside the bubble where is near the liquid film. In the case of velocity contour at the liquid film in the streamwise direction, the velocity gradually increases as the location changes to the rear direction as shown in Fig. 5(a). This increase in the relative velocity may influence the drag reduction on the wall.

\section{SUMMARY AND CONCLUSIONS}

We investigated large bubble behavior in wall turbulent flow of a horizontal channel flow. It can be achieved by the basis of the previous study, and the previous study was observed the characteristics of a large bubble such as bubble shape and vortical structure around the bubble. To investigate these features, the present work was visualized in two parts; inside of the bubble for understanding bubble shape and the liquid film to investigate the mechanism of generation of vortical structure. As a result, some vortexes were observed from inside of the bubble, the instantaneous velocity from the pair of vortices maintains the bubble shape like a plate. In the case of the liquid film, the bubble-induced spanwise velocity was generated, and this became the source to generate a vortical structure and persisted to the wake. 
(a)
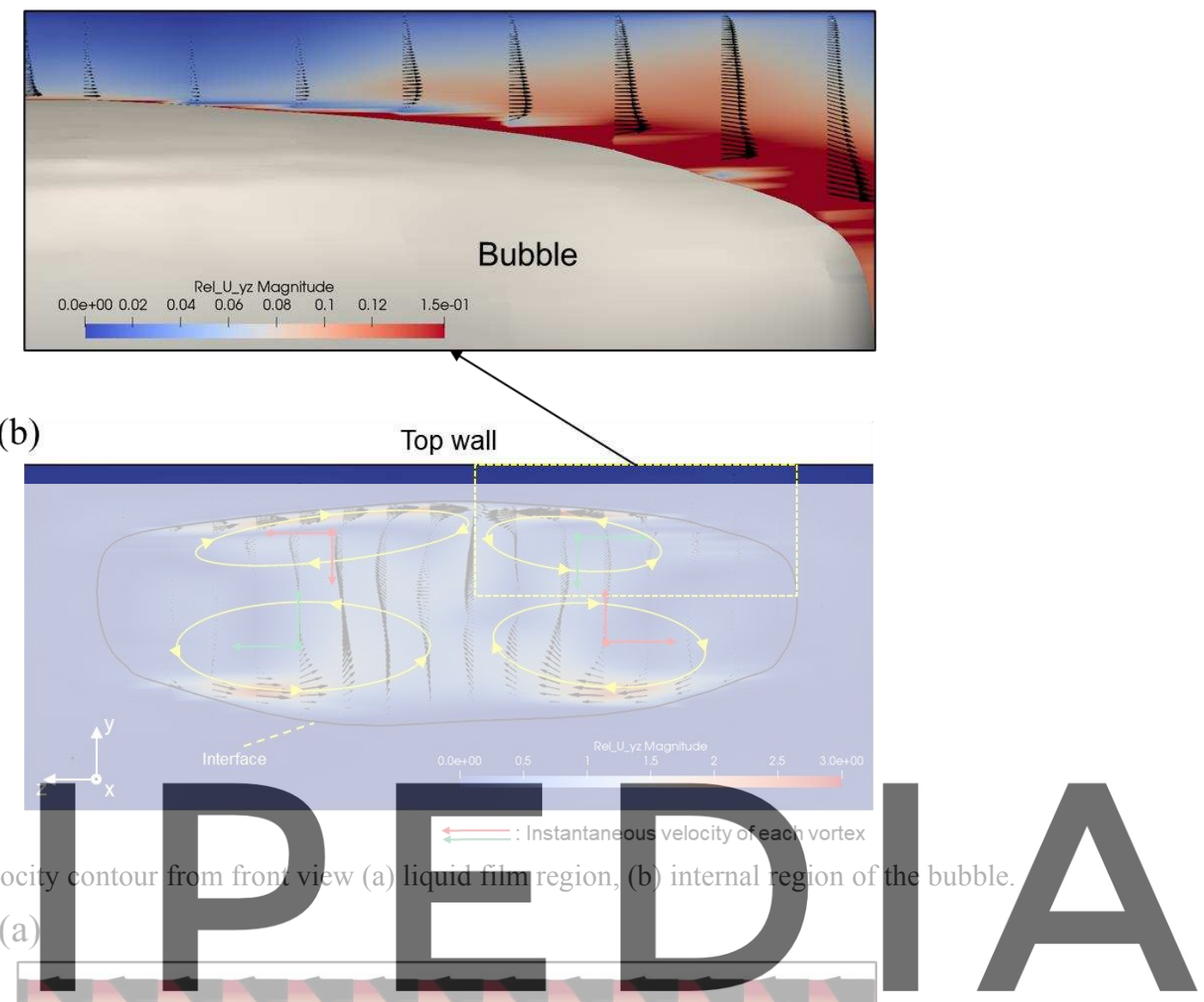

Register for free at https//www.scipedia.com to download the version without the watermark Bubble
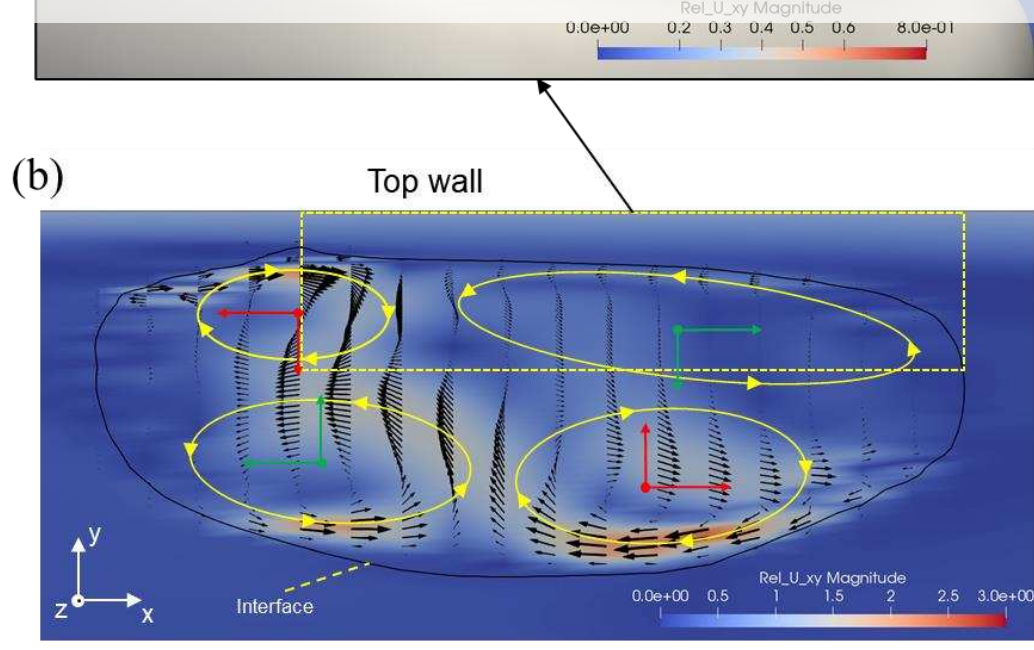

$\leftleftarrows$ : Instantaneous velocity of each vortex

Figure 5: Velocity contour from side view (a) liquid film region, (b) internal region of the bubble. 


\section{ACKNOWLEDGEMENTS}

This Research and development work was supported by the MEXT Doctoral program for Data-Related InnoVation Expert Hokkaido University (D-DRIVE-HU) program and highperformance computing project (hp 190113); the Fundamental Research Development Association for Shipbuilding and Offshore (REDAS); and the F3 Engineering Education and Research Center, Faculty of Engineering, Hokkaido University.

\section{REFERENCES}

[1] Kim, S.W., Oshima, N., Murai, Y., Park, H.J., Numerical investigation of a single intermediate-sized bubble in horizontal turbulent channel flow, J. Fluid Sci. Technol. Vol. 15, Issue 3, (2020)

[2] American Bureau of Shipping. Air Lubrication Technology. (2019):1-15

[3] McCormick, M. and Bhattacharyya, R. Drag reduction of a submersible hull by electrolysis, Nav. Eng. J. (1973) 85 (2): 11-16.

[4] Fukuda, K., Tokunaga, J., Nobunaga, T., Nakatani, T., Iwasaki, T. and Kunitake, Y. Frictional Drag Reduction with Air Lubricant over Super Water Repellent Surface (2nd Report) -Resistance Tests of Tanker and High Length to beam ratio Ship Models-, Journal of the Society of Naval Architects of Japan, Vol. 1999, Issue 186, (1999).

[5] Fukuda, K., Tokunaga, J., Nobunaga, T., Nakatani, T. and Iwasaki, T. Frictional drag reduction with air lubricant over a super-water-repellent surface, J. Marine Sci. Tech., 5,
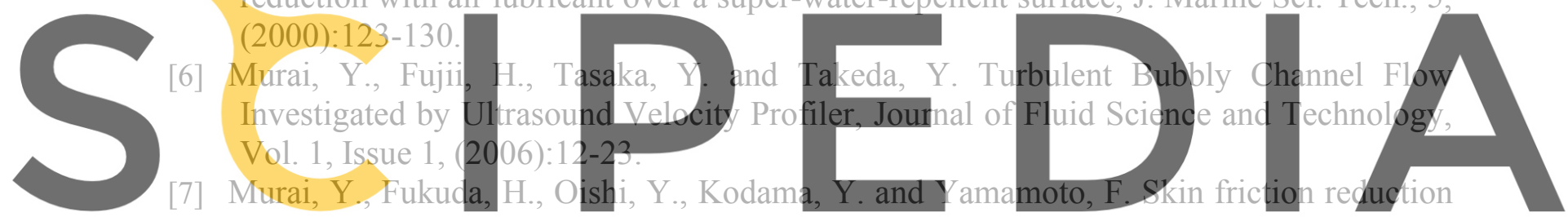

by large air bubbles in a horizontal channel flow, International Journal of Multiphase

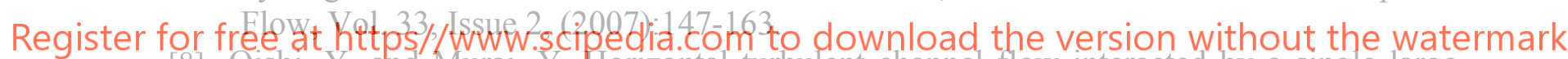

[8] Oishi, Y. and Mura1, Y. Horizontal turbulent channel flow interacted by a single large bubble, Experimental Thermal and Fluid Science Vol. 55, (2014):128-139.

[9] Gamet, L., Scala, M., Roenby, J., Scheufler, H., Pierson, J-. L., Validation of volume-offluid OpenFOAM ${ }^{\circledR}$ isoAdvector solvers using single bubble benchmarks, Comput. Fluids 213, 104722. 